\title{
Incorporation of Fluorotryptophan into Triostin Antibiotics by Streptomyces triostinicus
}

\author{
By A. CORNISH, ${ }^{1}$ K. R. FOX,${ }^{1}$ S. SANTIKARN, ${ }^{2} M . J$. WARING ${ }^{1 *}$ AND \\ D. H. WILLIAMS ${ }^{2}$ \\ 'Unicersity of Cambridge Department of Pharmacology, Medical School, Hills Road, \\ Cambridge CB2 2QD, UK \\ 'University Chemical Laboratory, Lensfield Road, Cambridge CB2 IEW, UK
}

(Received 15 August 1984; revised 29 October 1984)

\begin{abstract}
The quinoxaline chromophores of the antibiotics produced by Streptomyces triostinicus are derived from tryptophan. Protoplasts of this organism made novel products when they were incubated with DL-5-fluorotryptophan or DL-6-fluorotryptophan. When added to batch cultures of the organism, DL-5-fluorotryptophan, at concentrations as low as $10 \mu \mathrm{M}$, inhibited both mycelial growth and triostin production, but gave rise to novel products. These have been characterized, using fast atom bombardment mass spectrometry, as novel triostins in which one or both of the quinoxaline rings contain an atom of fluorine. The chromatographic properties of the triostins arising from the incorporation of DL-5-fluorotryptophan are very similar to those of triostins containing chlorine or bromine at position 6 of the quinoxaline ring; they are clearly different from those having a chlorine atom at position 7. Accordingly, it is suggested that the carbon atom at position 5 of the indole ring of tryptophan ends up at position 6 of the quinoxaline ring system in triostins $\mathrm{A}$ and $\mathrm{C}$.
\end{abstract}

\section{INTRODUCTION}

Triostin antibiotics are cyclic octadepsipeptides produced by several Streptomyces species (Kuroya et al., 1961; Katagiri, 1972). They are characterized by the possession of two quinoxaline-2-carboxylic acid chromophores and a disulphide cross-bridge (Otsuka \& Shoji, 1965) (Fig. 1). There are three naturally-occurring members of the family which differ only in the nature of the branched-chain amino acid residues at two rotationally equivalent positions in the peptide ring (Fig. 1).

The triostins have potent antitumour and antimicrobial activity (Katagiri et al., 1975) which may be attributed to their capacity to bind to the DNA of susceptible cells via a mechanism involving simultaneous intercalation of both chromophores (Waring, 1979). Using a series of chromophore-substituted derivatives it has been shown that the quinoxaline rings play an important role in determining the antimicrobial activities and DNA-binding properties of these antibiotics (Cornish et al., 1983a). To date, novel triostins have been prepared by a process of directed biosynthesis which exploits the finding that the producing organisms will incorporate into the antibiotics a variety of heterocyclic acids (Yoshida et al., 1968, 1970; Katagiri et al., 1975; Cornish et al., 1983a) in place of the natural chromophore precursor, which appears to be quinoxaline-2-carboxylic acid (Yoshida et al., 1970).

As regards the production of triostins by Streptomyces sp. s-2-210L, it has been shown that all the amino acid residues of the peptide ring are derived from the corresponding L-isomers, the $\mathrm{N}$ methyl groups coming from methionine (Yoshida \& Katagiri, 1969). By means of isotopic labelling studies it has been shown that this organism converts tryptophan to quinoxaline-2carboxylic acid, probably via L-kynurenine and kynuramine (Yoshida et al., 1970). 




Fig. 1. The structure of triostins. Triostin $\mathrm{A}, \mathrm{R}_{1}=\mathrm{R}_{2}=\mathrm{L}-N$-methylvaline; triostin $\mathrm{C}, \mathrm{R}_{1}=\mathrm{R}_{2}=\mathrm{L}-N, \gamma-$ dimethyl-allo-isoleucine; triostin $\mathbf{B}_{0}, \mathrm{R}_{1}=\mathrm{L}-N$-methylvaline, $\mathrm{R}_{2}=\mathrm{L}-N, \gamma$-dimethyl-allo-isoleucine.

The present study began with an investigation into the origin of the quinoxaline chromophores of the triostins produced by Streptomyces triostinicus; it then turned into a feasibility study to examine whether chromophore-substituted triostins might be produced by supplementing cultures with analogues of tryptophan rather than analogues of quinoxaline-2-carboxylic acid. Protoplasts of $S$. triostinicus were used as tools to examine the short-term effects of substituted tryptophans on triostin biosynthesis and to screen for incorporation into novel antibiotics. Conventional cultures of mycelia were used to prepare new derivatives in quantities sufficient for characterization by fast atom bombardment mass spectrometry.

\section{METHODS}

Chemicals. Solvents and general reagents were purchased from Fisons, Loughborough, Leicestershire, UK. All derivatives, isomers and analogues of tryptophan were obtained from Sigma. Quinoxaline-2-carboxylic acid was a gift from ICI. Halogenated derivatives of quinoxaline-2-carboxylic acid were obtained from the same sources as previously reported (Cornish et al., 1983a). Triostin A, triostin C and derivatives of triostin A having both quinoxaline chromophores replaced by 7-chloroquinoxaline-2-carboxyl, 6-chloroquinoxaline-2-carboxyl, or 6bromoquinoxaline-2-carboxyl residues were prepared and characterized as previously described (Cornish et al., $1983 a)$.

Radiochemicals. The following ${ }^{1+} \mathrm{C}$-labelled amino acids were purchased from Amersham: DL-[methylene${ }^{1+} \mathrm{C}$ ]tryptophan, $56.5 \mathrm{mCi} \mathrm{mmol}^{-1}$; DL-[benzene ring $\left.{ }^{1+} \mathrm{C}\right]$ tryptophan, $79 \mathrm{mCi} \mathrm{mmol}^{-1}$; $\mathrm{L}^{-}\left[\right.$methyl $\left.^{-1+} \mathrm{C}\right]$ methionine, $56.7 \mathrm{mCi} \mathrm{mmol}^{-1}: \mathrm{L}-\left[\mathrm{U}^{-1+} \mathrm{C}\right]$ alanine, $171 \mathrm{mCi} \mathrm{mmol}^{-1} ; \mathrm{L}-[\mathrm{U}-1+\mathrm{C}]$ valine, $285 \mathrm{mCi} \mathrm{mmol}^{-1} ; \mathrm{L}^{-}\left[\mathrm{U}^{-1+} \mathrm{C}\right] \mathrm{cysteine}$, $30 \cdot 2 \mathrm{mCi} \mathrm{mmol}^{-1}$; and $\mathrm{L}-\left[\mathrm{U}^{-1+} \mathrm{C}\right]$ serine, $170 \mathrm{mCi} \mathrm{mmol}^{-1}(1 \mathrm{mCi}=37 \mathrm{MBq})$. These isotopically labelled materials were used at the specific activities supplied unless otherwise stated.

Organism and culture conditions. Streptomyces triostinicus ATCC 21043 (a gift from ICI) was maintained on ISP7 agar (Shirling \& Gottlieb, 1966). Spore suspensions were prepared from two slants and used to inoculate a 2-litre Erlenmeyer flask containing $500 \mathrm{ml}$ of the seed medium described by Yoshida \& Katagiri (1967). Mycelium which had developed after $48 \mathrm{~h}$ incubation in this medium provided the inoculum for subsequent experiments. All incubations were done in a New Brunswick G-25 orbital incubator operating at $28^{\circ} \mathrm{C}$ and 240 r.p.m.

Preparation of protoplasts. The susceptibility of mycelia of $S$. triostinicus towards lysozyme was found to be critically dependent on the composition of the medium used to support growth. After some experimentation the following medium was devised which yielded satisfactory results: D-glucose, $20 \mathrm{~g}$; Bacto-peptone, $10 \mathrm{~g}$; yeast extract, $10 \mathrm{~g} ; \mathrm{NaCl}, 3 \mathrm{~g} ; \mathrm{CaCO}_{3}, 3 \mathrm{~g}$; and deionized water, 1 litre ( $\mathrm{pH} 7 \cdot 0$ ). Protoplasts were prepared from mycelia grown in 2-litre Erlenmeyer flasks containing $500 \mathrm{ml}$ of this medium. Each flask was inoculated with $20 \mathrm{ml}$ of culture prepared in the seed medium (see above). After $72 \mathrm{~h}$ incubation, the mycelium from a single flask was harvested by centrifugation ( $1000 \mathrm{~g}$ for $15 \mathrm{~min}$ ), washed once with $500 \mathrm{ml} 10 \%(\mathrm{w} / \mathrm{v})$ sucrose, collected on a tared filter paper using a Buchner funnel and the wet weight determined. The mycelial cake was resuspended in the protoplast buffer of Keller \& Kleinkauf (1977) $\left[25 \mathrm{mM}\right.$-TES; $25 \mathrm{mM}-\mathrm{CaCl}_{2} ; 10 \mathrm{mM}-\mathrm{MgCl}_{2} ; 0 \cdot 3 \mathrm{mM}-\mathrm{K}_{2} \mathrm{HPO}_{4}$; $0 \cdot 3 \mathrm{M}$-sucrose; and D-galactose, $10 \mathrm{~g} \mathrm{l}^{-1}\left(\mathrm{pH}^{7 \cdot 6)}\right)$ to a density of $1 \mathrm{~g}$ cells per $5 \mathrm{ml}$ buffer. Solid lysozyme [40000 
units (mg protein $)^{-1}$ ] was added to the mycelial suspension to a concentration of $2.5 \mathrm{mg} \mathrm{ml}^{-1}$ and protoplasts were prepared as follows (based on the method of Cornish et al., 1983 ). The suspension was incubated in a New Brunswick Aquatherm water bath operating at $28^{\circ} \mathrm{C}$ and 100 r.p.m. Release of protoplasts was monitored as described by Keller \& Kleinkauf (1977) and found to be complete $135 \mathrm{~min}$ after exposure of the mycelium to lysozyme. Residual intact mycelium was removed by passing the incubation mixture through a column $(10 \times 2.5 \mathrm{~cm})$ containing two plugs of tightly packed glass wool. The filtrate was then centrifuged at $5000 \mathrm{~g}$ for $25 \mathrm{~min}$ at room temperature and the resulting protoplast pellet was resuspended in 3-5 $\mathrm{ml}$ of fresh buffer and filtered through two more layers of tightly packed glass wool. The $\mathrm{OD}_{650}$ of the suspensions was measured at an appropriate dilution in a Unicam SP500 spectrophotometer, and was adjusted by the addition of protoplast buffer to give a value of $8 \cdot 0$. Protoplasts were then used immediately for biosynthetic studies. To check that incorporation of precursors could not be attributed to the persistence of intact mycelia, the level of triostin synthesis (see below) was measured using protoplast suspensions in which the sucrose concentration had been diluted to $0 \cdot 1 \mathrm{M}$ or less. In these tests the level of incorporation never exceeded $4 \%$ of the values obtained with suspensions containing $0.3 \mathrm{M}-$ sucrose.

Biosinthesis of triostin by protoplasts. Incubations were started by adding $0.1 \mathrm{ml}$ volumes of protoplast suspension $\left(O D_{n 50}=8.0\right)$ to $0.1 \mathrm{ml}$ protoplast buffer containing an appropriate ${ }^{1+} \mathrm{C}$-labelled precursor of the triostin molecule together with the particular analogue/metabolite of tryptophan under investigation. The quantities of radioactivity used and the concentrations of analogues of tryptophan are given in Results. Protoplast suspensions were shaken in flat-bottomed tubes $(10 \times 2.5 \mathrm{~cm})$ mounted vertically in a New Brunswick Aquatherm water bath operating at $28{ }^{\circ} \mathrm{C}$ and 110 r.p.m. Incubations were stopped after $120 \mathrm{~min}$ by the addition of $5 \mathrm{ml}$ ice-cold deionized water. Triostins were extracted into $5 \mathrm{ml}$ ethyl acetate. The organic extracts were taken to dryness, redissolved in chloroform and loaded on to plastic-backed TLC sheets (Merck Kieselgel 60F $254,0.2 \mathrm{~mm}$ thickness) which were then developed using ethyl methyl ketone. Radiolabelled products were located using autoradiography. The extent of incorporation of the ${ }^{1+} \mathrm{C}$-label into triostins was measured using liquid scintillation counting: appropriate regions of the TLC sheets were cut out and immersed for counting silica-side uppermost in $10 \mathrm{ml}$ toluene containing $0.4 \%(\mathrm{w} / \mathrm{v})$ butyl-PBD. Counting efficiencies with this system lay between 85 and $88 \%$.

Effects of tryptophan analogues on the production of triostins in batch culture. S. triostinicus was grown in 1-litre Erlenmeyer flasks containing $500 \mathrm{ml}$ of the maltose minimal medium of Yoshida \& Katagiri (1969) previously shown to support triostin production by this organism (Katagiri, 1972; Cornish et al., 1983a). Flasks were inoculated with $12.5 \mathrm{ml}$ volumes of seed culture. Tryptophan analogues, dissolved in a small volume of ethanol, were added into cultures at the time of inoculation. Each day, $10 \mathrm{ml}$ volumes were withdrawn and the mycelium collected on Whatman $\mathrm{GF} / \mathrm{C}$ filters $(2.5 \mathrm{~cm}$ diameter $)$ using vacuum filtration. The mycelium was extracted twice using $10 \mathrm{ml}$ volumes of acetone each time, and the residue was then dried and weighed to provide a measure of growth. The levels of triostin in the acetone extracts were determined spectrophotometrically as previously described (Cornish et al., 1983a).

HPLC. The antibiotic composition of acetone extracts was examined using HPLC. Extracts were first passed through Millipore FGLD 01300 filters and then $20 \mu$ l volumes were injected on to a Chrompack $C_{18}$ reversephase column $(25 \mathrm{~cm})$. Elution was done at ambient temperature using acetonitrile/water $(1: 1, \mathrm{v} / \mathrm{v})$ at a flow rate of $2.0 \mathrm{ml} \mathrm{min}-1$. The effluent was monitored at $243 \mathrm{~nm}$.

Fast atom hombardment mass spectrometry. Antibiotic mixtures as well as pure compounds were analysed by this technique, as described by Santikarn et al. (1983).

Purification of a notel dericatice of triostin A from cultures supplemented with DL-5-fluorotryptophan. Cultures of S. triostinicus were grown in two 2-litre flasks each containing 1 litre of the maltose minimal medium of Yoshida \& Katagiri (1969). The cultures were supplemented with DL-5-fluorotryptophan $(50 \mu \mathrm{M})$ at the time of inoculation. The mycelium was harvested for extraction of antibiotics after $9 \mathrm{~d}$ incubation as previously described (Cornish $e t$ al., 1983a). The major novel triostin was purified using flash column chromatography (Still et al., 1978) followed by preparative TLC as described by Cornish et al. (1983a);6 mg of the novel derivative were recovered.

\section{RESULTS}

\section{Synthesis of triostins de novo by protoplasts of $S$. triostinicus}

Fig. 2 shows radiolabelled products recovered from suspensions of protoplasts incubated with those ${ }^{14} \mathrm{C}$-labelled amino acids previously reported by Yoshida \& Katagiri (1969) to provide the entire carbon skeleton of triostin A in Streptomyces sp. s-2-210L. Two of the labelled products were identified as triostins $A$ and $C$. With the exception of $\mathrm{L}-\left[\mathrm{U}-{ }^{14} \mathrm{C}\right]$ valine, each ${ }^{14} \mathrm{C}$-labelled amino acid was incorporated into triostins $A$ and $C$ in a similar ratio (Table 1).

The predominant labelling of triostin A with $\mathrm{L}-\left[\mathrm{U}-{ }^{14} \mathrm{C}\right]$ valine was as expected, since this antibiotic contains two residues of L- $N$-methylvaline presumably derived directly from this 


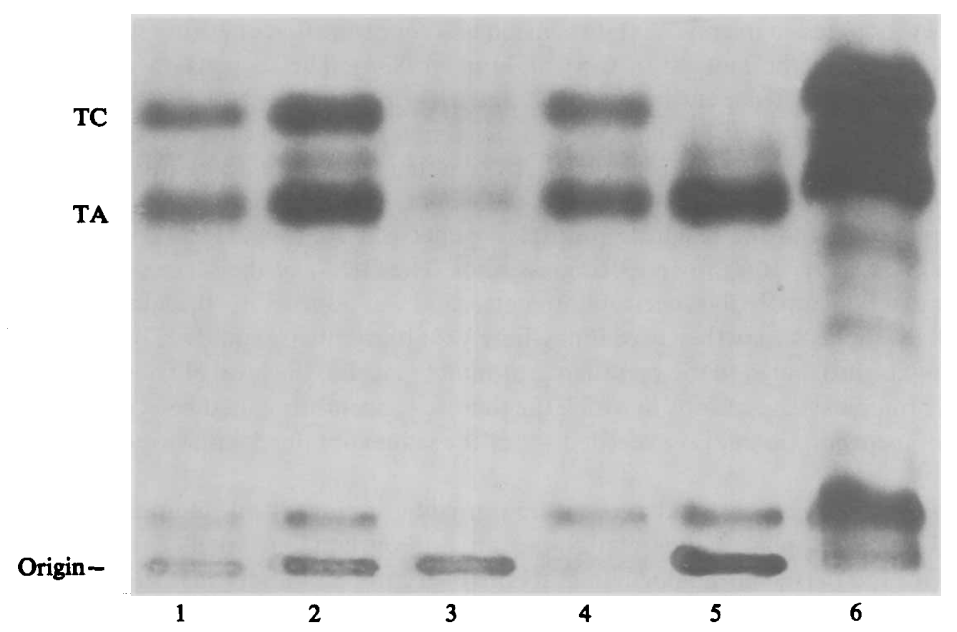

Fig. 2. Incorporation of ${ }^{1+} \mathrm{C}$-labelled amino acids into triostins by protoplasts. The autoradiograph shows labelled products recovered from protoplast suspensions $\left(0.2 \mathrm{ml}, \mathrm{OD}_{650}=4.0\right)$ which had been incubated for $2 \mathrm{~h}$ with ${ }^{1+} \mathrm{C}$-labelled amino acids : track 1, DL-[benzene ring-U-14 C]tryptophan $(0 \cdot 25 \mu \mathrm{Ci})$; track 2, L- $\left[\mathrm{U}-{ }^{-1+C}\right]$ serine $(1.0 \mu \mathrm{Ci})$; track $3, \mathrm{~L}-[\mathrm{U}-1+\mathrm{C}]$ alanine $(1.0 \mu \mathrm{Ci})$; track $4, \mathrm{~L}-\left[\mathrm{U}-{ }^{14} \mathrm{C}\right]$ cysteine $(1.0 \mu \mathrm{Ci}) ; \operatorname{track} 5, \mathrm{~L}-\left[\mathrm{U}-{ }^{1+} \mathrm{C}\right]$ valine $(1.0 \mu \mathrm{Ci})$; track $6, \mathrm{~L}-\left[\right.$ methyl $\left.{ }^{-1+} \mathrm{C}\right]$ methionine $(0.5 \mu \mathrm{Ci})$. At the end of the incubation, the protoplasts were lysed and antibiotics extracted as described in Methods. Standards of triostin A (TA) and triostin C (TC) were added to each extract and were located by illuminating the chromatograms with UV light at $254 \mathrm{~nm}$. Labelled products were detected by autoradiography.

amino acid (Yoshida \& Katagiri, 1969), whereas in triostin C these residues are replaced by two moieties of $\mathrm{L}-N, \gamma$-dimethyl-allo-isoleucine (Otsuka \& Shoji, 1965). A minor component was always detected running on chromatograms between triostins $\mathbf{A}$ and $\mathrm{C}$. This was probably triostin $\mathrm{B}_{0}$, a hybrid structure containing one residue of $\mathrm{L}-N$-methylvaline and one of $\mathrm{L}-N, \gamma-$ dimethyl-allo-isoleucine, which is often produced in small amounts by these organisms (Katagiri, 1972). Other minor radioactively labelled components (compare also Fig. 3) may well be precursors of the natural antibiotics (I. E. Swift \& M. J. Waring, unpublished observations). Their identity and metabolic properties are currently under investigation.

The fact that very little label from $\mathrm{L}-\left[\mathrm{U}-{ }^{14} \mathrm{C}\right]$ valine was recovered in triostin $\mathrm{C}$ shows that the carbon skeleton of this amino acid was not distributed appreciably amongst other constituents of the antibiotics, though this possibility was not investigated for the other ${ }^{14} \mathrm{C}$-labelled precursors.

\section{Effect of quinoxaline-2-carboxylic acid on the labelling of triostins by ${ }^{14} \mathrm{C}$-labelled tryptophan}

From the data in Table 2 it can be seen that addition of quinoxaline-2-carboxylic acid markedly reduced the extent of incorporation of DL-[benzene ring-U-14 C]tryptophan and DL[methylene ${ }^{-14} \mathrm{C}$ ]tryptophan into triostins $\mathrm{A}$ and $\mathrm{C}$. As expected a large reduction in the extent of labelling was also observed by adding L-tryptophan (Table 2 ) while no effect was found with Dtryptophan, even when this was present in incubations at a concentration of $10 \mathrm{~mm}$ (results not shown). Addition of quinoxaline-2-carboxylic acid did not reduce the extent of labelling of triostins by $\mathrm{L}-\left[\right.$ methyl- $\left.{ }^{14} \mathrm{C}\right]$ methionine; indeed it led to a $56 \%$ stimulation of incorporation. Evidently quinoxaline-2-carboxylic acid was competing directly with ${ }^{14} \mathrm{C}$-labelled tryptophan, and not inhibiting antibiotic synthesis in a non-specific fashion.

\section{Effects of analogues of quinoxaline-2-carboxylic acid and analogues of tryptophan on the synthesis of triostins by protoplasts}

Suspensions of protoplasts behaved in all respects like cultures of $S$. triostinicus as regards their capacity to make new products in the presence of 6-chloroquinoxaline-2-carboxylic acid (and its methyl ester), 7-chloroquinoxaline-2-carboxylic acid, and 6-bromoquinoxaline-2- 

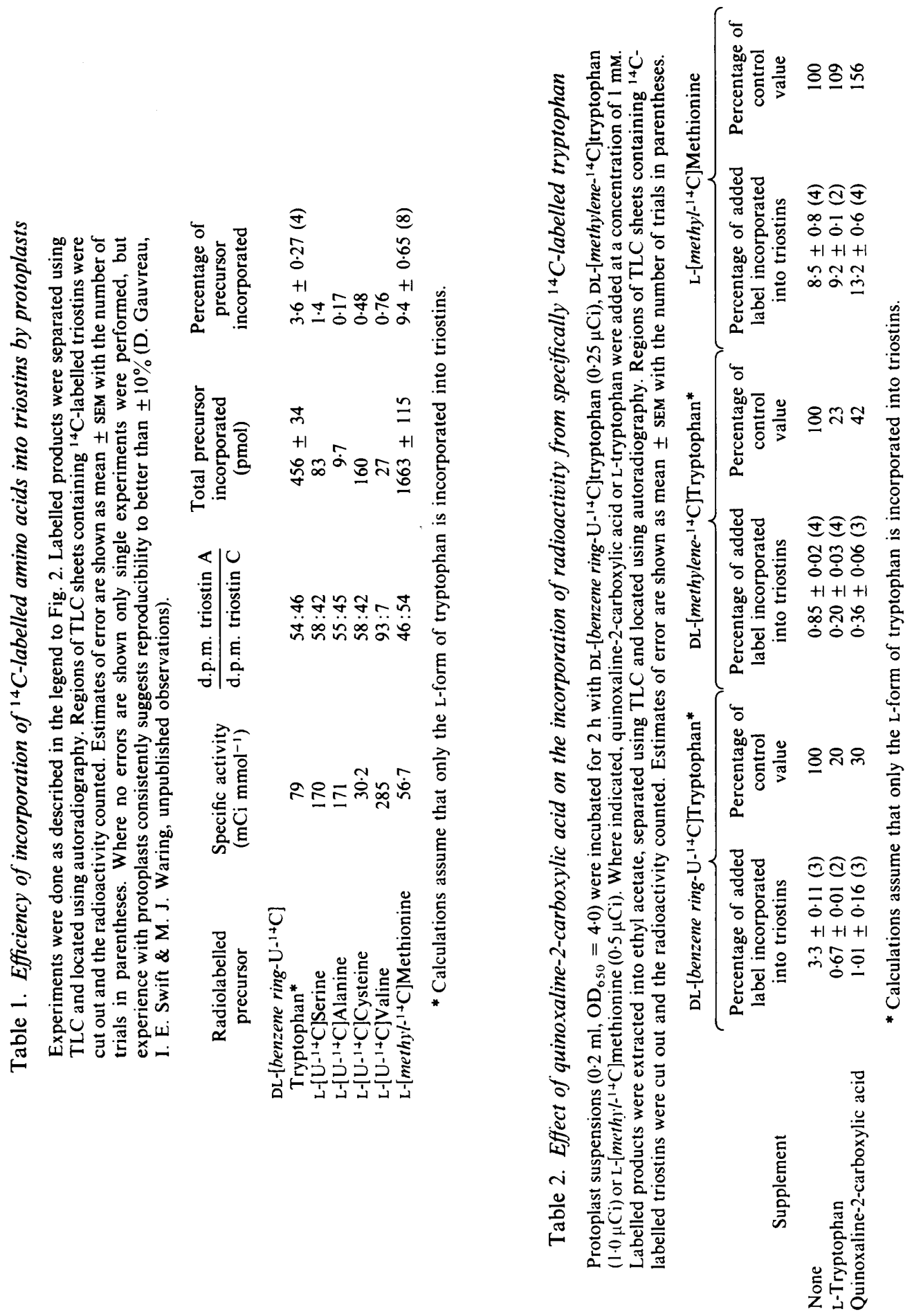


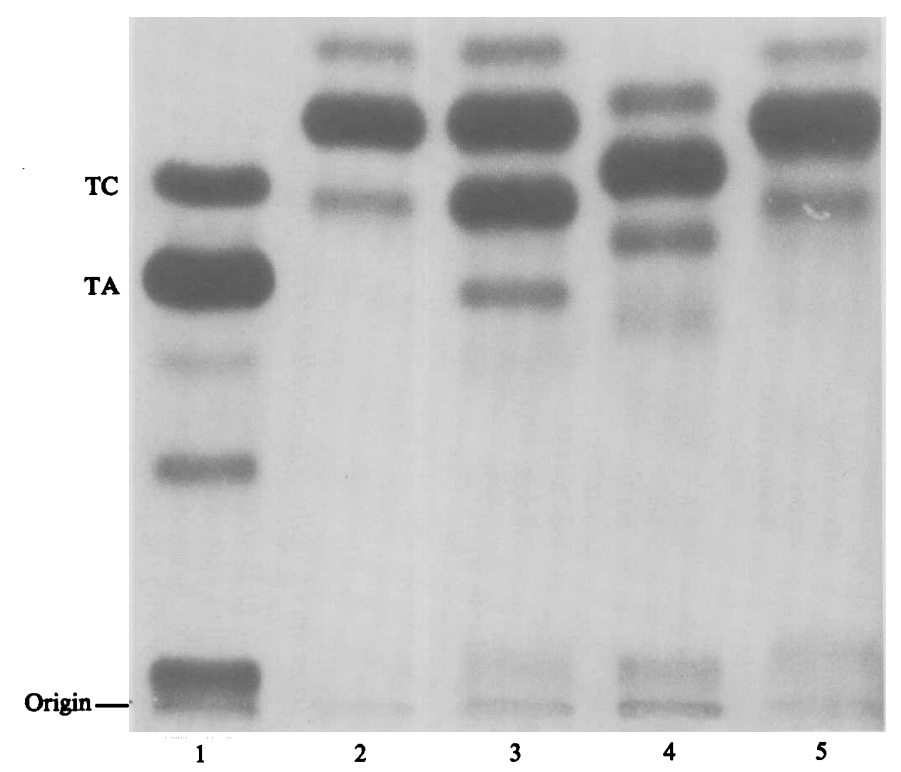

Fig. 3. Production of novel triostins by protoplast suspensions incubated with analogues of quinoxaline-2-carboxylic acid. The autoradiograph, taken from a TLC sheet, shows labelled products recovered from protoplast suspensions $\left(0.2 \mathrm{ml}, \mathrm{OD}_{650}=4.0\right)$ which had been incubated for $2 \mathrm{~h}$ with $\mathrm{L}^{-}$ [methy ${ }^{1+}+\mathrm{C}$ ]methionine $(0.5 \mu \mathrm{Ci})$ and analogues of quinoxaline-2-carboxylic acid $(1 \mathrm{mM}):$ track 1 , quinoxaline-2-carboxylic acid; track 2, 6-chloroquinoxaline-2-carboxylic acid; track 3, the methyl ester of 6-chloroquinoxaline-2-carboxylic acid; track 4, 7-chloroquinoxaline-2-carboxylic acid; track 5, 6bromoquinoxaline-2-carboxylic acid. The positions of standards of triostin A (TA) and triostin C (TC) are indicated.

carboxylic acid (Cornish et al., 1983a) (Fig. 3). Cultures of $S$. triostinicus incorporate these analogues into novel triostins, giving rise to a mixture of six antibiotics containing triostin $\mathrm{A}$, triostin $C$ (the levels of which are markedly reduced relative to unsupplemented controls) and their respective derivatives in which one or both quinoxaline chromophores are replaced (Cornish et al., 1983a). All six components may be resolved using HPLC, but complete separation has not proved possible using TLC. From Fig. 3 it can be seen that all four halogenated derivatives of quinoxaline-2-carboxylic acid markedly suppressed synthesis of the natural triostins by protoplasts, and led to the appearance of two or three additional labelled bands in each case. In order of increasing chromatographic mobility, these correspond to the mono-substituted derivative of triostin A, the bis-substituted derivative of triostin A migrating closely with the mono-substituted derivative of triostin $\mathrm{C}$, and the bis-substituted derivative of triostin $\mathrm{C}$. In each experiment the major novel, labelled component comigrated with a standard of the respective bis-substituted derivative of triostin A. It is clear from Fig. 3 that the chromatographic mobilities of triostins having a halogen atom at position 6 of the quinoxaline ring (tracks 2, 3 and 5) are very different from those of derivatives having a substituent at position 7 (track 4). This shows that the quinoxaline rings are important determinants of chromatographic mobility, and that it is possible to distinguish between triostins having halogen atoms at positions 6 or 7 of the ring system on the basis of their relative mobilities.

The autoradiograph in Fig. 4 shows products labelled by $\mathrm{L}-\left[\right.$ methyl $\left.{ }^{-14} \mathrm{C}\right]$ methionine recovered from suspensions of protoplasts which had been incubated with racemic mixtures of 4-, 5- or 6fluorotryptophan, or 5-, 6- or 7-methyltryptophan. New labelled products were detected only with DL-5-fluorotryptophan and DL-6-fluorotryptophan. The chromatographic mobilities of the new compounds made with DL-5-fluorotryptophan were very similar to those of triostins having a chlorine or bromine atom at position 6 of the quinoxaline ring (compare Figs 3 and 4). By contrast, the novel products made with DL-6-fluorotryptophan migrated similarly to triostins having a chlorine atom at position 7 of the ring system. These results suggested that $S$. triostinicus 


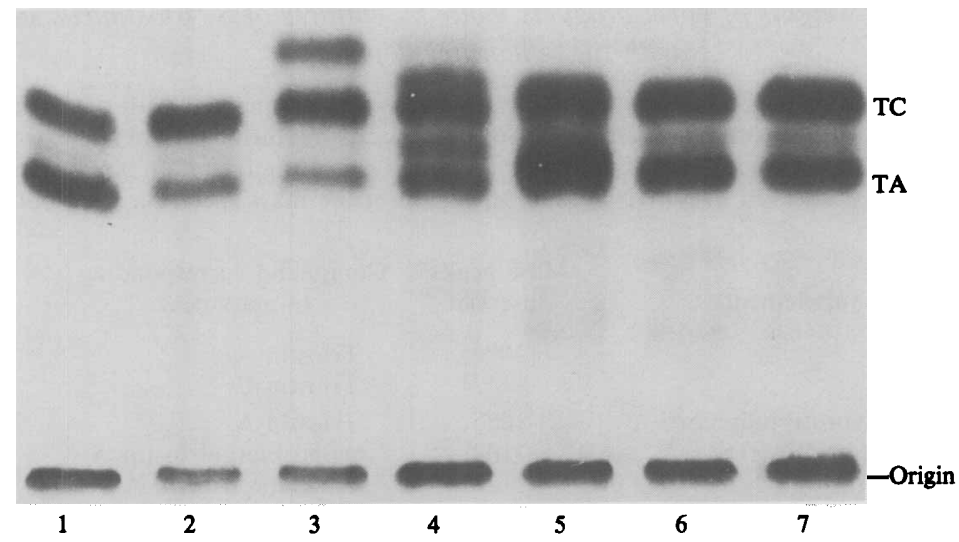

Fig. 4. Effects of analogues of tryptophan on the synthesis of triostins by protoplasts. The autoradiograph, taken from a TLC sheet, shows labelled products recovered from suspensions of protoplasts $\left(0.2 \mathrm{ml}, \mathrm{OD}_{650}=4 \cdot 0\right)$ which had been incubated for $2 \mathrm{~h}$ with L-[methyl- $\left.{ }^{1+} \mathrm{C}\right]$ methionine $(0 \cdot 5$ $\mu \mathrm{Ci})$ plus analogues of tryptophan $(1 \mathrm{mM})$ : track 1 , control (unsupplemented); track 2, DL-4fluorotryptophan; track 3, DL-5-fluorotryptophan; track 4, DL-6-fluorotryptophan; track 5, DL-5methyltryptophan; track 6, DL-6-methyltryptophan; track 7, DL-7-methyltryptophan. The positions of standards of triostin A (TA) and triostin C (TC) are indicated.

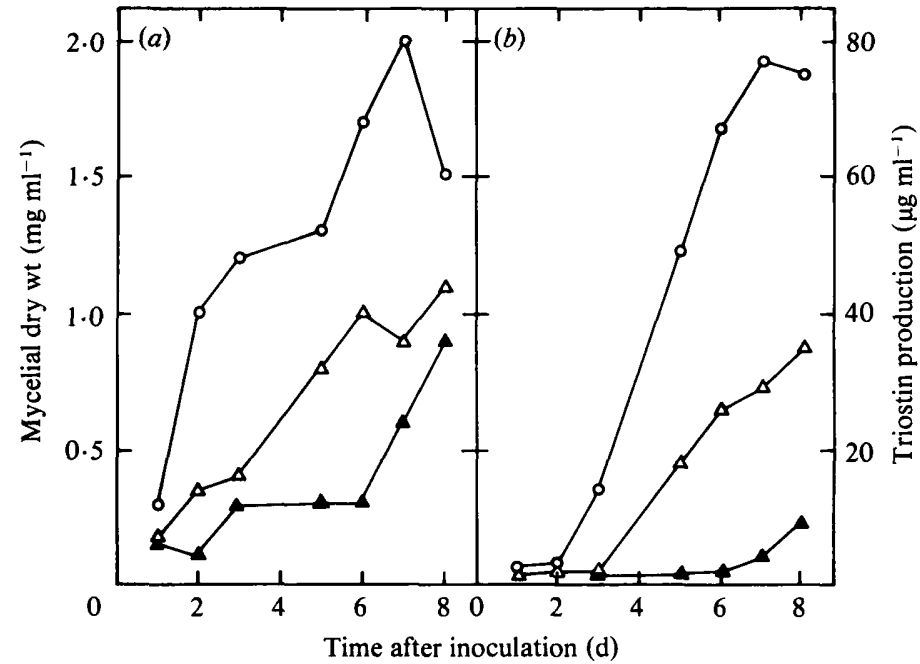

Fig. 5. Effect of DL-5-fluorotryptophan on mycelial growth $(a)$ and antibiotic production $(b)$. $S$. triostinicus was grown in 1-litre flasks containing $500 \mathrm{ml}$ of maltose minimal medium. The amino acid analogue was added to cultures at the time of inoculation. $O$, Control; $\triangle, 10 \mu \mathrm{M}$-5-fluorotryptophan;

$\Delta, 50 \mu \mathrm{M}$-5-fluorotryptophan.

is able to incorporate 5- and 6-fluorinated derivatives of tryptophan into triostins, the fluorine atoms ending up at positions 6 and 7 of the heterocyclic ring system, respectively. Neither of these analogues of tryptophan suppressed production of the natural antibiotics to the extent observed with analogues of quinoxaline-2-carboxylic acid (compare Figs 3 and 4).

\section{Effect of DL-5-fluorotryptophan on the production of triostins by batch cultures of $S$. triostinicus}

Further experiments were undertaken to investigate whether the novel products were made by mycelia over a longer period of time and to characterize them further. DL-5-fluorotryptophan inhibited growth and antibiotic production at concentrations as low as $10 \mu \mathrm{M}$ (Fig. 5) whereas DL-tryptophan had little or no effect, even at an initial concentration of $1 \mathrm{~mm}$ (not shown). 
Table 3. Molecular weights of novel products made by cultures of $S$. triostinicus supplemented with DL-5-fluorotryptophan

The organism was grown in $500 \mathrm{ml}$ maltose minimal medium supplemented with DL-5-fluorotryptophan $(10 \mu \mathrm{M}$ or $50 \mu \mathrm{M})$ at the time of inoculation. After $8 \mathrm{~d}$ incubation the mycelium was extracted with acetone as described in Methods, and the extracts were analysed using fast atom bombardment mass spectrometry. Peaks observed in the 1000-1200 $M_{\mathrm{r}}$ region of the mass spectrum were noted.

$\begin{array}{ccl}\text { Supplement } & \begin{array}{c}\text { Mass peaks } \\ \text { observed }\end{array} & \begin{array}{c}\text { Compound corresponding } \\ \text { to mass peak }\end{array} \\ \text { None } & 1086 & \text { Triostin A } \\ \text { DL-5-Fluorotryptophan } & 1142 & \text { Triostin C } \\ (10 \mu \mathrm{M} \text { or } 50 \mu \mathrm{M}) & 1086 & \text { Triostin A } \\ & 1104 & \text { mono-Fluorotriostin A } \\ & 1122 & \text { bis-Fluorotriostin A } \\ & 1142 & \text { Triostin C } \\ 1160 & \text { mono-Fluorotriostin C } \\ 1178 & \text { bis-Fluorotriostin C }\end{array}$

Extracts of cultures supplemented with DL-5-fluorotryptophan at concentrations of $10 \mu \mathrm{M}$ and $50 \mu \mathrm{M}$ were analysed by HPLC and found to contain four components in addition to triostins $\mathrm{A}$ and $C$.

When extracts of unsupplemented cultures were examined by means of fast atom bombardment mass spectrometry, abundant molecular ions were detected which corresponded to triostins A and C (mol. wts 1086 and 1142, respectively). Extracts of cultures supplemented with DL-5-fluorotryptophan gave rise to four additional peaks in the mass spectrum at molecular weights expected for the respective mono- and bis-fluorine-substituted derivatives of triostins A and $\mathrm{C}$ (Table 3). The major novel component produced by cultures containing $50 \mu \mathrm{M}-\mathrm{DL}-5-$ fluorotryptophan was purified as a white powder and found to have a molecular weight of 1122 , which is correct for bis-fluoroquinoxaline triostin A (Table 3).

\section{DISCUSSION}

The results presented here establish that L-tryptophan is readily incorporated into triostins by $S$. triostinicus, and that chromophore-substituted antibiotic analogues may be produced by supplementing cultures with derivatives of tryptophan as well as with derivatives of quinoxaline-2-carboxylic acid. Substituted tryptophan compounds have also been used to prepare analogues of pyrrolnitrin (Gorman et al., 1968) and indolmycin (Werner \& Demain, 1981), although both of these are comparatively small antibiotics which are derived wholly (pyrrolnitrin) or partially (indolmycin) from tryptophan.

Yoshida et al. (1970) proposed that Streptomyces sp. s-2-210L converts tryptophan to quinoxaline carboxylic acid by way of L-kynurenine and kynuramine (Fig. 6a). Original evidence for the existence of the pathway depicted in Fig. $6 a$ was obtained from experiments in which mycelial suspensions of Streptomyces sp. s-2-210L were incubated with samples of $\left[{ }^{1+} \mathrm{C}\right]$ tryptophan specifically labelled at known positions in both the indole ring system and the alanyl side-chain. The incorporation of the label into the quinoxaline chromophores of triostin was subsequently determined (Yoshida et al., 1970). However, the exact fate of individual carbon atoms in the benzene ring of tryptophan was not investigated. Besides examining the feasibility of producing novel triostins from substituted tryptophans, one of the reasons for the present study was to discover whether relatively inexpensive, commercially available analogues of tryptophan could be used to reveal the fate of particular aromatic carbon atoms of tryptophan upon its conversion into the quinoxaline ring system. We have clearly shown that DL-5fluorotryptophan is incorporated to produce fluorotriostins, but it has not yet proved possible to determine unambiguously the precise location of the fluorine atoms in the quinoxaline ring system of the novel antibiotics. However, comparison of their chromatographic mobilities with those of halogenated derivatives previously characterized (Cornish et al., 1983a; Santikarn et 


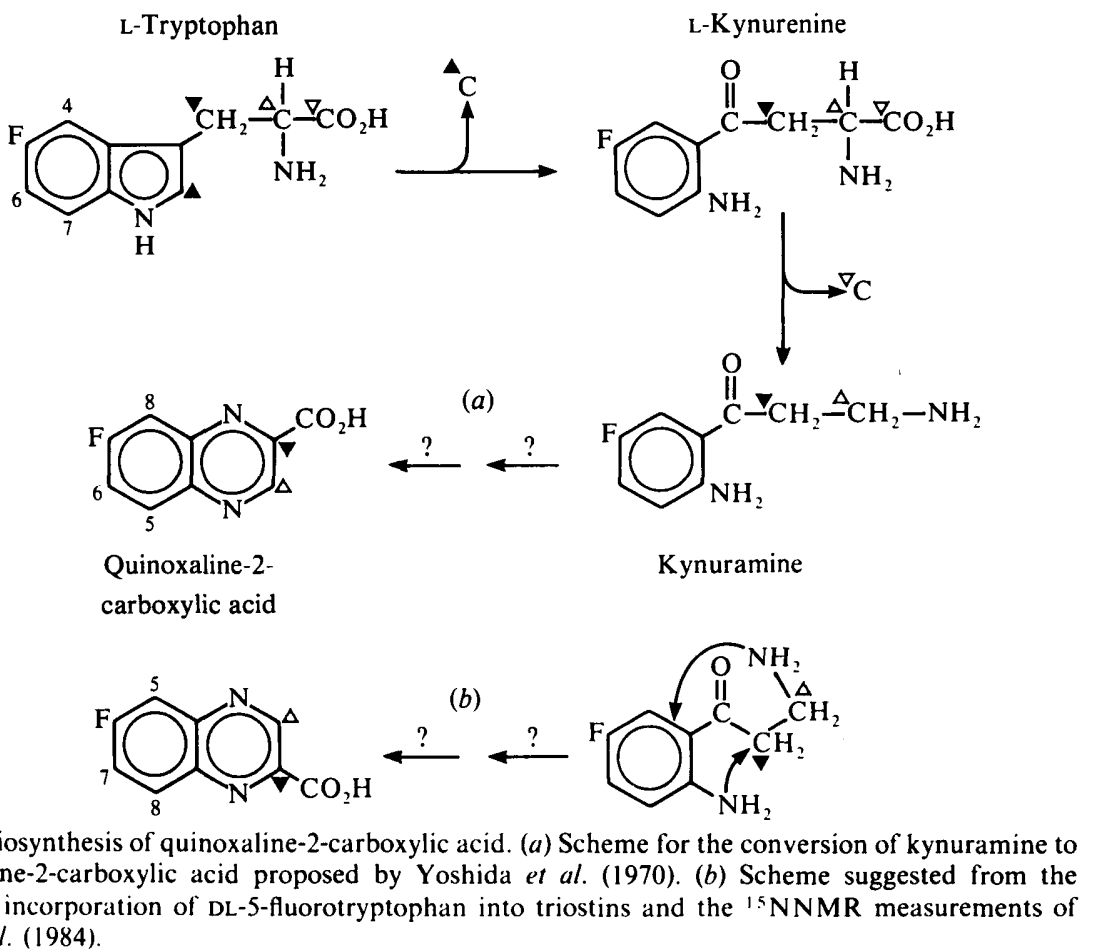

Fig. 6. Biosynthesis of quinoxaline-2-carboxylic acid. (a) Scheme for the conversion of kynuramine to quinoxaline-2-carboxylic acid proposed by Yoshida et al. (1970). (b) Scheme suggested from the observed incorporation of DL-5-fluorotryptophan into triostins and the ${ }^{15}$ NNMR measurements of Reid et al. (1984).

al., 1983) suggests that the fluorine atom at position 5 of the tryptophan ring system ends up at position 6 in quinoxaline-2-carboxylic acid, whereas the scheme for the conversion of tryptophan to quinoxaline-2-carboxylic acid advanced by Yoshida et al. (1970) predicts that it should end up at position 7 (Fig. 6a). A plausible alternative mechanism which is consistent with both the results of previous labelling studies and the findings reported here is given in Fig. 6(b). In this scheme, both nitrogen atoms of tryptophan would be incorporated into quinoxaline-2carboxylic acid, the indole giving rise to $\mathrm{N}(1)$ of the quinoxaline ring system and the amino group giving rise to N(4) (Fig. 6 b). The origin of the nitrogen atoms in the quinoxaline ring system of triostins has recently been investigated independently using ${ }^{15} \mathrm{~N}$ NMR spectroscopy, with results which are entirely consistent with the mechanism depicted in Fig. 6(b) (Reid et al., 1984).

In previous work we reported that derivatives of quinoxaline-2-carboxylic acid which are incorporated into triostins by $S$. triostinicus may be added into cultures at an initial concentration of $1 \mathrm{~mm}$ without affecting the total level of antibiotic production (Cornish et al., 1983a). By contrast, DL-5-fluorotryptophan proved inhibitory at an initial concentration as low as $10 \mu \mathrm{M}$. This difference no doubt arises because tryptophan is an intermediate in a number of metabolic pathways essential for growth of the organism, whereas quinoxaline-2-carboxylic acid is an end-product of metabolism needed only for triostin synthesis. Incorporation of analogues of quinoxaline-2-carboxylic acid into triostins surely results from the broad substrate specificity of the enzyme(s) responsible for attaching the chromophores to the peptide ring. Accordingly, the failure to observe incorporation of methyltryptophan into triostins probably reflects the more restricted substrate specificity of enzymes involved in the conversion of tryptophan to quinoxaline-2-carboxylic acid. Probably this will ultimately limit the range and number of novel triostins which can be produced from substituted derivatives of tryptophan.

This work was supported by grants from the Medical Research Council, the Royal Society, and the Cancer Research Campaign. A.C. thanks the Science and Engineering Research Council for a CASE award sponsored and materially aided by the authorities of ICI Pharmaceuticals Division, Alderley Park, Macclesfield, Cheshire. We acknowledge the able technical assistance of Mrs T. Douglas. 


\section{REFERENCES}

Cornish, A., Fox, K. R. \& Waring, M. J. (1983a). Preparation and DNA-binding properties of substituted triostin antibiotics. Antimicrobial Agents and Chemotherapy 23, 221-231.

Cornish, A., Waring, M. J. \& Nolan, R. D. (1983b). Conversion of triostins to quinomycins by protoplasts of Streptomyces echinatus. Journal of Antibiotics 36, $1664-1670$.

Gorman, M., Hamill, R. L., Elander, R. P. \& Mabe, J. (1968). Preparation of substituted phenyl pyrroles through the metabolism of tryptophan analogues. Biochemical and Biophysical Research Communications 31, 294-298.

KATAGIRI, K. (1972). Method for producing triostin. US Patent no. 3647631.

Katagiri, K., Yoshida, T. \& Sato, K. (1975). Quinoxaline antibiotics. In Antibiotics III. Mechanism of Action of Antimicrobial and Antitumour Agents, pp. 234-251. Berlin: Springer-Verlag.

Keller, V. \& Kleinkauf, H. (1977). Studies of the biosynthesis of actinomycin in protoplasts from Streptomyces antibioticus. Archires of Biochemistry and Biophysics 184, 111-124.

Kuroya, M., Ishida, N., Katagiri, K., Shoji, J., Yoshida, T., Mayama, M., Sato, K., Matsuura, S., Ninomi, Y. \& Shiratori, O. (1961). Studies on quinoxaline antibiotics. I. General properties and the producing strains. Journal of Antibiotics A14, 324-329.

Otsuka, H. \& SHOJ, J. (1965). The structure of triostin C. Tetrahedron 21, 2931-2938.

Reid, D. G., Doddrell, D. M., Williams, D. H. \& Fox, K. R. (1984). A ${ }^{15} \mathrm{~N}$ nuclear magnetic resonance study of the biosynthesis of quinoxaline antibiotics. Biochimica et hiophysica acta 798, 111-114.
SANTIKaRN, S., Hammond, S. J., Williams, D. H., CORnish, A. \& WARING, M. J. (1983). Characterization of novel antibiotics of the triostin group by fast atom bombardment mass spectrometry. Journal of Antibiotics 36, 362-364.

Shirling, E. B. \& Gottlieb, D. (1966). Methods for characterization of Streptomyces species. International Journal of Systematic Bacteriology 16, 313340.

Still, W. C., Khan, M. \& Mitra, A. (1978). Rapid chromatographic technique for preparative separations with moderate resolution. Journal of Organic Chemistry 43, 2923-2925.

Yoshida, T. \& KaTAGIRI, K. (1967). Influence of isoleucine upon quinomycin biosynthesis. Journal of Bacteriology. 93, 1327-1331.

Yoshida, T. \& KaTAGIRI, K. (1969). Biosynthesis of the quinoxaline antibiotic, triostin, by Streptomyces s-2-210L. Biochemistry 8, 2645-2651.

Yoshida, T., Kimura, Y. \& Katagiri, K. (1968). Novel quinomycins. Biosynthetic replacement of the chromophores. Journal of Antibiotics 21, 465-467.

Yoshida, T., Kimura, Y. \& KatagiRI, K. (1970). The biosynthesis of the quinoxaline antibiotic, triostin, by Streptomyces s-2-210L. On the role of quinoxaline2-carboxylic acid. In Progress in Antimicrobial and Anticancer Chemotherapy, vol. 2, pp. 1160-1165. Tokyo: University of Tokyo Press.

WARING, M. J. (1979). Echinomycin, triostin and related antibiotics. In Antibiotics V, Part II. Mechanism of Action of Antimicrobial and Antitumour Agents, pp. 234-251. Berlin: Springer-Verlag.

Werner, R. G. \& Demain, A. L. (1981). Directed biosynthesis of new indolmycins. Journal of Antibiotics 34, 551-554. 